\title{
Evolution of External and Internal Implant to Abutment Connection
}

\author{
Ninad Muley, DR Prithviraj, Vikas Gupta
}

\begin{abstract}
A study of the implant to abutment connection is of paramount importance as it is the primary determinant of the strength and stability of the implant supported restoration, which in turn determines the restoration's prosthetic stability. Traditionally, the Branemark's external hexagon has been widely used but its significant complications like abutment screw loosening, rotational misfit at implant-abutment interface and microbial penetration have led to modification of the external hexagon and the development of the internal implant-abutment connections. This review describes the various implantabutment connections that have evolved overtime from the traditional external hexagon.
\end{abstract}

Keywords: Implant-abutment interface, External hexagon, Internal hexagon, Spline dental implants, Morse taper implants.

How to cite this article: Muley N, Prithviraj DR, Gupta V. Evolution of External and Internal Implant to Abutment Connection. Int J Oral Implantol Clin Res 2012;3(3):122-129.

\section{Source of support: Nil}

Conflict of interest: None

\section{INTRODUCTION}

The roots of implant dentistry were laid way back in the 1980's in the United States of America in the Branemark's protocol. Since then, implant dentistry has continuously evolved from the original Branemark protocol to include varied techniques and applications., ${ }^{1,2}$ This evolution has been possible because numerous investigators have documented the biological factors, surgical procedures and restorative principles that influence the outcome of implant restorations, thus widening the applications of implant dentistry from restoration of a single tooth to multiple missing teeth with predictable success. ${ }^{3,4}$ The mechanical principles governing implant restorations have also been clearly defined and understood. ${ }^{5,6}$ Improvement in restorative principles and better understanding of the perceived outcome of implant therapy has led to the development of the concept of restoration-driven implant dentistry. ${ }^{7}$

The original Branemark's protocol involves a two-stage surgical procedure and was designed to restore a completely edentulous mandibular arch. ${ }^{8}$ The first step involved the placement of a titanium screw into viable bone and an undisturbed healing period of at least 3 months. The next step involved the exposure of the implant, attachment of a transmucosal element and the connection of the implant to the prosthetic component of the restoration. In this protocol, the implant-abutment interface was an external hexagon of $0.7 \mathrm{~mm}$ height. This external hex served the purpose of a torque transfer coupling device (fixture mount) during the initial placement of the implant into the bone and the subsequent connection of the transmucosal extension, which when used in series could effectively restore the completely edentulous arch. Although the external hex served the aforementioned purposes, it was not an effective antirotation device $^{9}$ and was not designed to withstand the forces directed on the crowns intraorally. ${ }^{10}$ These properties are required when implants are used to restore partially edentulous arches or a single missing tooth. Thus, implant manufacturers had to compensate for this by changing the type of screw used (e.g. geometry, height, surface area), the precision of the fit over the hex, and the amount of torque used to secure the screws. ${ }^{11-13}$

Also, to overcome the inherent deficiencies of the original external hex, a variety of implant to abutment connections have evolved from it. The goals of new designs are to improve connection stability throughout the placement and function and simplify the armamentarium necessary for the clinician to complete the restoration. The implantabutment interface determines joint strength, stability, and lateral and rotational stability of the joint. ${ }^{14}$ Thus, the joint stability is one of the most important parameters for the success of implant therapy.

A number of implant-abutment connection designs are commercially available and the clinician is often perplexed as to which implant system and which connection design to choose. This literature review analyses the evolution of various implant-abutment connections from the traditional external hexagon implant to the morse taper implants and aims to provide the clinician with an overview of the various commercially available implant-abutment connections.

\section{SEARCH STRATEGY}

An electronic search was performed of articles on Medline from September 1983 to June 2012. Keywords, such as implant abutment interface, external hexagon implants, internal hexagon implants, morse taper implants, were used alone or in combination to search the database. The option of 'related articles' was also utilized. Finally, a search was performed of the references of review articles and the most relevant papers. 
The electronic search was supplemented by hand searching of the journals relevant to the field of implant dentistry such as: International Journal of Oral and Maxillofacial Implants, Journal of Oral Implantology and Clinical Oral Implants and related research.

\section{The Implant-Abutment Interface}

The implant/abutment connection, by convention, is generally described as an internal or external connection. These two implant-abutment connections can be distinguished by whether or not there exists an extension of a geometric figure above the body of the implant. In external connection implants, we observe a distinct projection external to the body of the implant (Fig. 1), whereas in internal connection implants the implant-abutment connection is recessed into the body of the implant (Fig. 2).

The connection can be further characterized as a slip-fit joint, where a slight space exists between the mating parts, and the connection is passive, or as a friction-fit joint, where no space exists between the mating components and the parts are literally forced together.

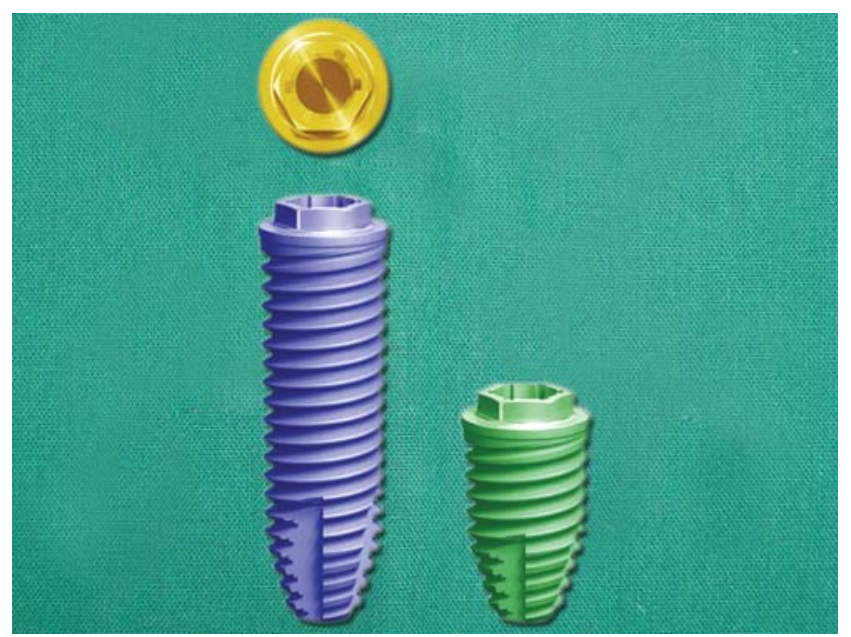

Fig. 1: The external implant to abutment connection

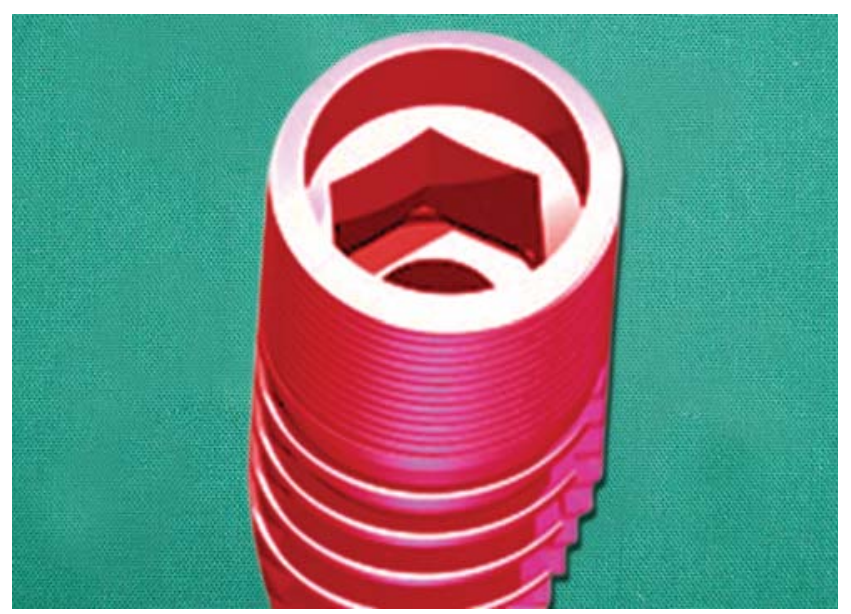

Fig. 2: The internal implant to abutment connection
The mating surfaces are further characterized as being a butt joint, which consists of two right-angled flat surfaces contacting one another, or a bevel joint, where the surfaces are angled either internally or externally. The joined surfaces may also incorporate a rotational resistance and indexing feature and/or lateral stabilizing geometry. This geometry is further described as octagonal, hexagonal, conical, cylinder hex and spline, etc.

\section{The External Implant-Abutment Connection}

The Branemark's original implant-abutment connection was a $0.7 \mathrm{~mm}$ external hexagon. The external hexagon served the purpose of coupling and as a torque transfer device. The original Branemark's protocol was developed for the restoration of completely edentulous arches using a series of implants connected to one another with a metal bar. ${ }^{8}$

The applications of implant dentistry have now expanded from the original restoration of completely edentulous arches to fixed partial dentures, single-tooth replacement, maxillofacial and a myriad of other applications, limited only by the ingenuity and skill of the clinician. ${ }^{15,16}$ Significant clinical complications of the Branemark's external hexagon connection ${ }^{9,17,18}$ makes it unsuitable for these applications. These complications include the incidence of abutment screw loosening and fracture, as well as dynamic micromotion at the implantabutment interface. ${ }^{19}$ Thus, the external hexagon has now evolved, by necessity, into a prosthetic indexing and antirotation mechanism.

The external hex since then has undergone a number of modifications and is now available in heights of $0.7,0.9$, 1.0 and $1.2 \mathrm{~mm}$ and with flat-to-flat widths of 2.0, 2.4, 2.7, 3.0, 3.3 and $3.4 \mathrm{~mm}$, depending on the implant platform. ${ }^{8}$ Also, a variety of modifications of the external hexagon, such as the tapered hexagon, external octagon and the spline dental implant ${ }^{20}$ are now available.

\section{Tapered Hexagon (Hex Lock Innovation)}

This design was developed with the aim of improving the fit between the implant and the abutment by incorporating a 1.5 degree taper to the hex flat and a corresponding closetolerance hexagonal abutment recess that is friction fitted onto the hex. It was first introduced by Swede-Vent TL (Paragon Implant Co, Encino, CA). A similar design is also available from the spectra implant system.

It was claimed by manufacturers to greatly reduce the rotational freedom between the implant and the abutment and thereby reduce the incidence of screw loosening.

By creating the tapered interface, the mating hexes interdigitate with frictional fit for added accuracy of the 
transfer procedure and increased stability in function. ${ }^{21}$ An independent study has proven the claim of zero micromotion at the implant-abutment interface of the tapered hexagon implant. $^{22}$ Thus, the tapered hexagon design of the implantabutment connection offers a definite advantage as compared to the traditional external hexagon.

\section{External Octagon}

The external octagon is an eight-sided external implantabutment connection. Commercially, it was first marketed as a 1-piece narrow diameter (3.3 and $3.5 \mathrm{~mm}$ ) implant (ITI Narrow Neck) designed for replacing the mandibular anterior teeth. The tall, octagonal extension allowed for 45 degree rotation. The manufacturers claimed the implant to be having good lateral, rotational resistance and strength, but relevant studies supporting this claim are not available.

Further, the tall external octagonal implant had a few disadvantages because of which it is not considered a very popular implant-abutment connection design. The tall external octagon is not compatible with the use of angled abutments. Further, the octagonal geometry of the implantabutment connection resembles a circle, and thus offers very little rotational resistance at the implant-abutment connection.

\section{Spline Dental Implant}

The spline dental implant ${ }^{20}$ system was developed by Calcitek (Calcitek, Carlsbad, CA) in the year 1992. The implant consists of six spline teeth which project outward from the body of the implant and fit into six grooves between the projections from the corresponding abutment. The series of opposing parallel splines match integrally with the corresponding grooves of the opposite member. The fit between the implant and the abutment is a 'snug fit' with excellent locational accuracy.

The spline dental implants are available in three different sizes. It is available in platform diameters of 4, 5 and $3.25 \mathrm{~mm} .^{20}$ The 4 and $5 \mathrm{~mm}$ diameter implants are strong and mechanically stable and offer the advantages of reduced incidence of screw loosening as well as minimal rotational movement as compared to the traditional external hexagon. The $3.25 \mathrm{~mm}$ diameter implant is thinner, with small spines and a narrow platform which result in a frail, vulnerable interface. No clinical reports have been published on the stability of this interface. Thus, though the 4 and $5 \mathrm{~mm}$ diameter spline dental implants have proven advantages over the traditional external hexagon, the $3.25 \mathrm{~mm}$ spline dental implant was not a very popular design.

Thus, a summary of the various external implant to abutment connections and their comparison to the traditional external hexagon has been provided in the Table 1.

\section{The Internal Implant-Abutment Connection}

To overcome the clinical complications with external implant-abutment connections, 9,17,18 internal connection implants were developed. The goals of new designs are to improve connection stability throughout function and placement and simplify the armamentarium necessary for the clinician to complete the restoration. One of the first internally hexed implants was designed with a $1.7 \mathrm{~mm}$-deep hex below a $0.5 \mathrm{~mm}$ wide, $45^{\circ}$ bevel. ${ }^{20,21}$ This was the corevent implant developed by Nickzick implant manufacturers in the year 1986. The design has been proven to distribute intraoral forces deep within the implant and thus improve the implant-abutment joint stability. ${ }^{23,24}$

Table 1: External implant to abutment connections

\begin{tabular}{|c|c|c|}
\hline S. no. & Type of external implant-abutment connection & Comparison with the traditional external hex \\
\hline 1. & Tapered hexagon & $\begin{array}{l}\text { Reduced freedom of rotation between the implant } \\
\text { and the abutment and thus reduced incidence of } \\
\text { screw loosening. } \\
\text { - Added accuracy of transfer procedure and added } \\
\text { stability in function. }\end{array}$ \\
\hline 2. & External octagon & $\begin{array}{l}\text { - It has the advantage of more number of positions } \\
\text { placing the abutment over the implant. } \\
\text { Due to the geometry of this connection representing } \\
\text { a circle, the connection has minimal resistance to } \\
\text { rotation and thus was not very popular. }\end{array}$ \\
\hline 3. & Spline dental implant & $\begin{array}{l}\text { Wider diameter spline dental implants have reduced } \\
\text { incidence of abutment screw loosening and reduced } \\
\text { rotational tendency. } \\
\text { - Narrow diameter spline dental implants lacked } \\
\text { stability and thus were not popular. }\end{array}$ \\
\hline
\end{tabular}


The internal interface designs offer a reduced vertical height platform for restorative components, distribution of lateral loading deep within the implant, a shielded abutment screw, long internal wall engagements that create a stiff, unified body that resists joint opening, wall engagement with the implant that buffers vibration, the potential for a microbial seal, extensive flexibility and the ability to lower the restorative interface to the implant level esthetically. ${ }^{8}$

The internal connection implants can be divided into the following groups for the purpose of explanation.

1. Passive fit/slip fit joint (space exists between mating components):

- 6-point internal hex:

- Center pulse-core vent/screw vent

- Friadent-Frialit-2

- 12-point internal hex

- 3i-osseotite certain

- 3-point internal tripod

- Alatech technologies, Camlog

- Nobel biocare/Replace select

- Internal octagon: Omniloc, Sulzer Calcitek

2. Friction fit (no space between mating components)

Locking taper/morse taper:

- 8 degree taper (ITI straumann, Avana, 3i TG, Ankylos)

- 11 degree taper (Astra)

- 1.5 degree tapered rounded channel (Bicon).

\section{6-Point Internal Hexagon Design}

This is the most common type of commercially available, internal implant-abutment connection. It has a six-sided geometric figure, that is, a hexagon recessed into the body of the implant. As the internal geometry is a hexagon, the abutment can fit over the implant at every 60 degree rotation of the implant over the abutment, but not at any other intermediate angle. Thus, abutment positioning is possible at six different positions of the implant over the abutment.

This type of implant-abutment connection is commercially available from various implant manufacturing companies. It is available from Central pulse (Screw-vent) implants with a $1.2 \mathrm{~mm}$ length of the internal connection. This implant has evolved from the original core vent implant, with a hollow basket design to the tapered screw vent implant. This new design is claimed by the manufacturer to simplify insertion and increases initial stability in soft bone by a patented surgical protocol of inserting the tapered implant into a straight socket. The design has been proven to distribute intraoral forces deep within the implant and thus improve the implant-abutment joint stability as compared to the traditional external hexagon. ${ }^{23,24}$
The internal hexagon connection is also commercially available from Friadent, Dentsply (Frialit-2). The Frialit-2 system is claimed to combine a cylindrical implant design with an internal connection. The cylindrical connection is claimed to provide lateral load resistance, resistance to joint opening, protection of abutment screw.

The basic design of the Frialit-2 system is that of a stepped cylinder. ${ }^{25}$ It has the advantage of distribution of axial as well as lateral loads effectively. Axial loads acting on the implant are distributed to the step plateaus, whereas lateral forces are dissipated to the enveloping surfaces. ${ }^{26}$ The internal hexagon connection provides a $60^{\circ}$ indexing and rotational resistance. Thus, this implant design combines the advantages of both a cylindrical design with an internal connection.

\section{2-Point Internal Hexagon}

The 12-point internal hexagon design, also marketed by some manufacturers as the offset hexagon design allows for greatest freedom of placement of the abutment over the implant. The 12-point double internal hex provides an opportunity to place the abutment on the implant for every 30 degree rotation, thus useful when we use angled abutments. It provides us with a greater opportunity to correct the off-axis angulation of the abutment with respect to the implant. One such implant is marketed by $3 \mathrm{i}$ Implant Innovations Inc., Palm Beach Gardens, Florida, which is the Osseotite Certain.

Though the 12-point internal hexagon design offers greater flexibility in the positioning of the abutment over the implant, the design should not compromise the mechanical properties of the implant-abutment interface. A study was conducted to investigate the mechanical characteristics of implant-abutment interface design in a dental implant system, using nonlinear finite element analysis (FEA) method. This finite element simulation study was applied on three commonly used commercial dental implant systems: The reduced-diameter 3i Implant System (West Palm Beach, FL, USA) with a hex and a 12-point double internal hexagonal connection, the Semados implant system (Bego, Bremen, Germany) with combination of a conical (45 taper) and internal hexagonal connection, the Brånemark implant system (Nobel Biocare, Gothenburg, Sweden) with external hexagonal connection. It was concluded that the reduced-diameter 3i Implant System with a hex and a 12-point double internal hexagonal connection had a better stress distribution and produced a smaller displacement than the other two implant systems. ${ }^{27}$ Thus, the 12-point internal hexagon system offers more freedom of positioning the abutment over the implant as well as a 
better distribution of stresses at the implant-abutment interface.

\section{3-Point Internal Tripod}

This type of implant to abutment connection represents a triangular internal geometry. A major disadvantage of this system is that it allows for positioning of the abutment over the implant at only 120 degree of rotation. This type of implant-abutment connection was introduced by Nobel Biocare, which was the replace select system. It represents the trichannel implant system. It is available in four diameters: 3.5, 4.3, 5 and $6 \mathrm{~mm}$ and is color-coded for ease of identification. As the replace select 3-point internal tripod system offers limited options for positioning of the implant over the abutment, it is not a very clinically preferred design. Further, a study conducted to evaluate the stress distribution at the implant-abutment interface of the replace select system showed that under off-axis loading the stress distribution was more favorable in the external hex connection as compared to the 12-point double hexagon connection. Thus, this is not a favorable implant-abutment connection. ${ }^{28}$

Camlog implant system (Alatech technologies) represents an internal tripod implant-abutment connection. The length of the internal connection is $5.4 \mathrm{~mm}$. It is claimed to have a 'tube in tube effect' which is claimed to provide an accurate, mechanically secure implant to abutment connection with antirotational stability.

\section{Internal Octagon Implant}

The internal octagonal implant represents an 8-sided internal geometry connecting the implant and the abutment allowing for positioning of the implant over the abutment at every 45 degree rotation. The internal octagon connection was introduced by Omniloc, Sulzer Calcitek. The octagonal connection, because of its thin walls, 0 to $6 \mathrm{~mm}$ length and a small diameter that presented a geometry profile similar to that of a circle, offered minimal rotational and lateral resistance during function. ${ }^{8}$ Due to these disadvantages, it is no longer marketed.

Before moving on to the friction fit or morse taper connections, a summary of the slip fit type of internal implant to abutment connections has been provided in Table 2.

\section{Friction Fit (Morse Taper Implants)}

The concept of morse taper implant-abutment connection design includes a tapered projection from the implant abutment, which fits into a tapered recess in the implant (Fig. 3). There is a friction fit and cold welding at the implant-abutment interface. This implant-abutment connection depends on this friction fit for elimination for rotation at the implant-abutment interface and subsequent abutment screw loosening. ${ }^{6}$

Sutter et al proposed a morse taper connection between implant and abutment as an optimal combination of predictable vertical positioning and self-locking characteristics. Similar results were reported by Norton who showed that the incorporation of conical connections between implant and abutment dramatically enhanced the ability of the system to resist bending forces. To date,

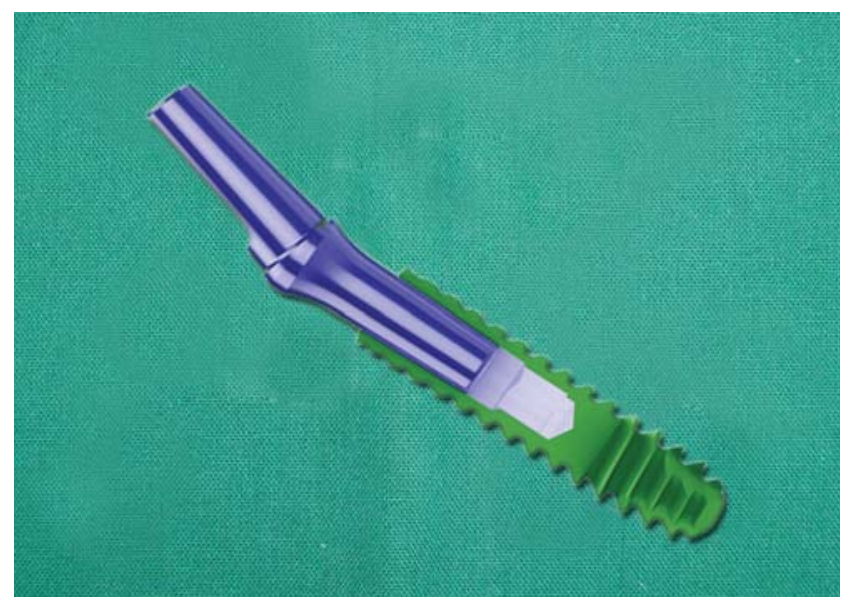

Fig. 3: The morse taper implant-abutment connection

Table 2: Internal implant to abutment connections: Slip/passive fit type

\begin{tabular}{|c|c|c|c|c|}
\hline S. no. & Type of connection & $\begin{array}{l}\text { Commercially } \\
\text { available implants }\end{array}$ & $\begin{array}{l}\text { Abutment positioning } \\
\text { over implant }\end{array}$ & $\begin{array}{l}\text { Number of } \\
\text { positions possible }\end{array}$ \\
\hline 1. & Internal hexagon & $\begin{array}{l}\text { Centerpulse-Core- } \\
\text { Vent/Screw-Vent } \\
\text { Friadent-Frialit-2 }\end{array}$ & $60^{\circ}$ & 6 \\
\hline 2. & 12-point hexagon & 3i Osseotite Certain & $30^{\circ}$ & 12 \\
\hline 3. & 3-point internal hexagon & $\begin{array}{l}\text { Alatech technologies: } \\
\text { Camlog, Nobel } \\
\text { Biocare: Replace select }\end{array}$ & $120^{\circ}$ & 3 \\
\hline 4. & Internal octagon & Omniloc, Sulzer Calcitek & $45^{\circ}$ & 4 \\
\hline
\end{tabular}


experiences published by Levine et al and Felton confirm this view. Studies have shown the other complications like the incidence of abutment screw loosening have also been reduced in the morse taper connection as compared to the external hex connection. ${ }^{6}$

The original concept of the morse taper included two tapers: 2 degree and 4 degree and is designed for a precise fit without self-locking thread.

\section{Degree Morse Taper Implants}

In dentistry, the concept of this morse taper or cone screw tapered connection was first utilized by the ITI group in Switzerland. ${ }^{29}$ The rationale was that a tapered connection would yield a mechanically stable, sound and self-locking interface. It basically creates a friction lock similar to the morse taper used in mechanical engineering and related industries.

A further modification of the ITI-Straumann implant design is the Synocta design. Though the original implant design allowed for a precise fit between the implant and the abutment, it did not allow for rotation of the abutment over the implant and fit at a different angulation. Wiskott and Belser supplemented the morse taper connection by introducing a internal hexagon in the middle of the morse taper connection, thus allowing for repositioning of the abutment and also precise transfer of the implant position to the master cast. As a result, only one transfer system and one analog are required.

A new implant introduced by Osteo-Ti, known as the Combi implant has a similar mechanism of accurate positioning and friction fit like the Synocta design and combines the features of an internal hex implant and a morse taper implant.

Other manufactures marketing the 8 degree morse taper implants are: Avana, 3i TG, Ankylos.

\subsection{Degree Morse Taper Implant}

This implant is marketed by Astra Tech. The fixture and abutment are strongly connected at an 11.5 degree angle by the conical seal design. The conical design seals off the connection and decreases micromovement and microleakage. This thread has a microthreaded conical neck and TiO blast surface. Microthreads on the fixture top prevent concentration of the stress around the alveolar ridge crest and decrease marginal bone loss.

\subsection{Degree Morse Taper Implants}

This is a true morse taper implant with an angle of taper: 1.5 degree is available from Bicon implants. The Bicon locking taper abutment has no screw, but like a screw- retained abutment, it relies on friction to keep it intact. Assembly is achieved by driving the 1.5 degree morse taper into the matching socket in the implant. A high clamping force between abutment and implant is generated by this action.

The high friction force is the result of relative slip between the two friction surfaces occurring at high contact pressure. This results in the surface oxide layers breaking down and causing cold welding at the implant-abutment interface.

\section{DISCUSSION}

This review describes the changes in the implant-abutment connection, from the $0.7 \mathrm{~mm}$ traditional external hexagon of the Branemark's implant and its various modifications, to the development of the morse taper type of implantabutment connections. The external hex connection used a coupling and torque transfer device in the Branemark's protocol, served adequately to restore the completely edentulous arch with a series of implants connected to one another with a metal bar. ${ }^{6}$ With better understanding of the concept of osseointegration and development and refinement of the surgical protocol in implant dentistry, the horizons of the applications of implant dentistry have widened.

Dental implants have now been used for a myriad of applications: From the restoration of the completely edentulous arch, to partial edentulous situations, single tooth replacements and fixed bridges. ${ }^{18,19}$ Thus, with the ever increasing applications of implant dentistry, the requirements of the implant-abutment connection have also increased. The implant-abutment connections must now serve the purpose of antirotation and prosthetic indexing. ${ }^{8}$ These functions are most important in the restoration of single posterior teeth by implants, as they are the most difficult to retain. ${ }^{30}$

Thus, there was a need to modify the Branemark's external hexagon implant-abutment connection to prevent complications, such as abutment screw loosening and fracture, which occurred commonly when the external hexagon was used in single tooth implant restorations. Thus, design modifications, such as increasing the height and flat to flat width of the mating surfaces of the implant-abutment connection have been tried by various manufacturers. ${ }^{8}$ The development of the tapered hexagon and the spline dental implant ${ }^{20}$ have been other attempts toward overcoming the limitations of the Branemark's external hexagon.

The search for a new implant-abutment connection design, to overcome the limitations of the external hexagon led to the development of the internal hexagon design. This has further been modified to the 3-point internal tripod, 
12-point internal hexagon and the internal octagon implantabutment connection. The basic clinical significance of these various implant-abutment connections is the freedom of positioning the abutment over the implant, which is maximum for the 12-point internal hex and minimum for the 3-point internal tripod. This review describes the various commercially available implant-abutment connections, highlights the claims of the manufacturers and supports it with published data wherever available.

Thus, this review aims at helping the clinician to make an informed decision as to which implant system and implant-abutment interface to use. It helps the clinician in making a choice from the array of commercially available implant-abutment interfaces.

\section{CONCLUSION}

The implant-abutment interface determines the lateral and rotational stability of the implant-abutment joint which in turn determines the prosthetic stability of the implantsupported restoration.

This review describes the evolution of the various implant-abutment connections and aims to inform the clinician regarding the various design characteristics associated with external and internal implant-abutment connections. It also enlightens the clinician about the clinical applications of the contemporary implant designs.

\section{DISCLOSURE}

The authors certify that they have no financial interest in any of the implant companies. As this is a review article not any research article, so there was no need of financial support. The authors report no conflict of interest.

\section{REFERENCES}

1. Lenchewski E. Esthetics and implant surgery. In: Aschheim KW, Dale BG (Eds). Esthetic dentistry: A clinical approach to techniques and materials (2nd ed). St. Louis, MO: Mosby 2001:429-40.

2. Salinas TJ, Sadan A. Establishing soft tissue integration with natural tooth-shaped abutments. Pract Periodont Aesthet Dent 1998;10:35-42.

3. Brånemark PI. Osseointegration and its experimental background. J Prosthet Dent 1983;50:399-410.

4. Avivi-Arber L, Zarb GA. Clinical effectiveness of implantsupported single-tooth replacement. The Toronto study. Int J Oral Maxillofac Impl 1996;1:311-21.

5. Khraisat A, Abu-Hammad O, Al-Kayed AM, Dar-Odeh N. Stability of implant abutment joint in a single tooth external hexagon system: Clinical and mechanical review. Clin Implant Dent Relat Res 2004;6:222-29.
6. Merz BR, Hunenbart S, Belser UC. Mechanics of the implantabutment connection: An 8-degree taper compared to a butt joint connection. Int J Oral Maxillofac Implants 2000;15:519-26.

7. Garber DA, Belser UC. Restoration-driven implant placement with restoration-generated site development. Compend Contin Educ Dent 1995;16(8):796-804.

8. Binon PP. Implants and components: Entering the new millennium. Int J Oral Maxillofac Implants 2000;15:76-94.

9. Beaty K. The role of screws in implant systems. Int J Oral Maxillofac Impl 1994;9(Spec Suppl):52-54.

10. Binon PP. Evaluation of machining accuracy and consistency of selected implants, standard abutments and laboratory analogs. Int J Prosthodont 1995;8:162-78.

11. Martin WC, Woody RD, Miller BH, Miller AW. Implant abutment screw rotations and preloads for four different screw materials and surfaces. J Prosthet Dent 2001 Jul;86:24-32.

12. Sakaguchi RL, Sun T, Haack JE. External strain distribution on implant prosthetic components. J Dent Res 1994;73:232.

13. Binon PP. The effect of implant/abutment hexagonal misfit on screw joint stability. Int J Prosthodont 1996;9:149-60.

14. Ohrnell L, Hirsh JM, Ericsson L, Brånemark PI. Single tooth rehabilitation using osseointegration. A modified surgical and prosthodontic approach. Quintessence Int 1988;19:871-76.

15. Jemt $\mathrm{T}$, Lekholm U. Oral implant treatment in posterior partially edentulous jaws: A 5-year follow-up study. Int J Oral Maxillofac Implants 1993;8:635-40.

16. Nevins $M$, Langer $B$. The successful application of osseointegrated implants to the posterior jaw: A long-term retrospective study. Int J Oral Maxillofac Implants 1993;8: 428-32.

17. English CE. Externally hexed implants, abutments and transfer devices: A comprehensive overview. Implant Dent 1992;1: 273-83.

18. Kallus T, Bessing C. Loose gold screws frequently occur in full arch fixed prostheses supported by osseointegrated implants after 5 years. Int J Oral Maxillofac Implants 1994;9:169-78.

19. Meng JC, Everts JE, Qian F, Gratton DG. Influence of connection geometry on dynamic micromotion at the implantabutment interface. Int J Prosthodont 2007:20;623-25.

20. Binon PP. The spline implant: Design, engineering and evaluation. Int J Prosthodont 1996:9;419-33.

21. Niznick G. The implant abutment connection: The key to prosthetic success. Compendium 1991 Dec;12(12):932-38.

22. Barzilay I. Rotational accuracy of implant components for single-tooth, root-form implants. Dental Implantology Update 1991;2(1):5-7.

23. Niznick GA. The Core-Vent ${ }^{\mathrm{TM}}$ implant system. The evolution of the osseointegration implant. Oral Health 1983;73:13-17.

24. Niznick GA. The implant abutment connection: The key to prosthetic success. Compend Cont Educ Dent 1991;12:932-37.

25. Krennmair G, Schmidinger S, Waldenberger O. Single-tooth replacement with the Frialit-2 system: A retrospective clinical analysis of 146 implants. Int J Oral Maxillofac Implants 2002;17:78-85.

26. d'Hoedt B. Dentale implantate aus polykristalliner Aluminiumoxidkeramik: Einheilung und Langzeitergebnisse [thesis]. Tübingen, Germany: University of Tübingen, 1991. 
27. Tang CB, Liu SY, Zhou GX, Yu JH, Zhang GD, Bao YD, et al. Nonlinear finite element analysis of three implant-abutment interface designs. Int J Oral Sci 2012;4(2):101-08.

28. Lan J, Xu X, Jiang G, Guan Y, Huang H, Liang J, et al. Threedimension finite element analyses of interior stress of two kinds of Replace implant. Hua Xi Kou Qiang Yi Xue Za Zhi 2011;29:464-68.

29. Ding TA, Woody RD, Higginbottom FL, Miller BH. Evaluation of the ITI Morse taper implant/abutment design with an internal modification. Int J Oral Maxillofac Implants 2003;18:865-72.

30. Misch CE, Misch-Dietsh F, Silc J, Barboza E, Cianciola LJ, Kazor C. Posterior implant single-tooth replacement and status of adjacent teeth during a 10-year period: A retrospective report. J Periodontol 2008;79:2378-82.

\section{ABOUT THE AUTHORS Ninad Muley (Corresponding Author)}

Postgraduate Student, Department of Prosthodontics, Government Dental College and Research Institute, Bengaluru, Karnataka, India e-mail: drninadmuley312@gmail.com

\section{DR Prithviraj}

Professor and Head, Department of Prosthodontics, Government Dental College and Research Institute, Bengaluru, Karnataka, India

\section{Vikas Gupta}

Postgraduate Student, Department of Prosthodontics, Government Dental College and Research Institute, Bengaluru, Karnataka, India 